\title{
NEUTRALIZED MICRO-DROPLET GENERATED BY ON-CHIP ELECTROHYDRODYNAMIC
}

\author{
Hang T. Nguyen ${ }^{1}$, Tung T. Bui ${ }^{1}$, Canh-Dung Tran ${ }^{2}$,Trinh D. Chu ${ }^{1}$, Hieu T. Vu ${ }^{3}$, Dzung V. Dao and \\ Van T. Dau ${ }^{3 *}$ \\ ${ }^{1}$ University of Engineering and Technology, Vietnam National University, Hanoi, VIETNAM \\ ${ }^{2}$ School of Mechanical and Electrical Engineering, University of Southern Queensland, Queensland \\ AUSTRALIA \\ ${ }^{3}$ School of Engineering and Built Environment, Griffith University, Queensland, AUSTRALIA
}

\begin{abstract}
A new conceptual design of electrospray has been developed to generate neutralized micro-droplet using onchip electro-hydrodynamic. A prototype of a bipolar electrostatic atomization with chambered nozzle tips has been carried out to demonstrate the capability of spaying viable, safe, neutral and alternative droplets which can be applied in micro/nano-encapsulation, bio-scaffold production and also the polymeric micro/nanoparticle fabrication over conventional fabrication techniques as well as the direct current electro-spraying/electrospinning. Several initially experimental results as presented through this work have shown the potential application of the present device in drug delivery.
\end{abstract}

\section{KEYWORDS}

Micro-droplet generation; electro-hydrodynamic; bipolar electrostatic atomization.

\section{INTRODUCTION}

Electrohydrodynamic atomization, also named as the electrospray (ES) method of fluids, occurs by a sufficiently strong electric field which acts on liquid moving in a capillary to induce the surface charge density. That yields a radial electrostatic pressure on the liquid surface equilibrated by the capillary pressure. Over the last many decades, this technique has been emerged as a powerful approach for biomedical applications, including drug delivery in respiratory treatment, drug discovery research and bio printing. Among the applications, the ES has currently attracted researchers to the inhalable drug delivery where the respiratory treatment consists of the generation and delivery of micro/nanoparticles as carriers for nanomedicine [1]. Together with the significant progress in the particle engineering and technology, the particle-based pulmonary delivery has emerged as a promising advance of the conventional inhalable dry powder or liquid drug formulations [2].

ES can be realized with various configurations. Among them, a typical nozzle-to-ring configuration consists of a nozzle as spray electrode and a ring as collector electrode. Under a relevantly high voltage, ES is generated at the nozzle tip and a flow of droplets moves toward the surface of its counter electrode. By the impact of ring electrode, most of the droplets are neutralized whereas a small part of them being highly charged can move through the ring owing to their inertia. In order to utilize the electrosprayed droplets for bio or health care applications, charge reduction to produce neutral clusters of selected compositions is imperative [3][4]. In this paper, a novel configuration has been developed using two spray electrodes which are symmetrically placed from each other and simply powered by a direct current power source

\section{CONCEPT AND DESIGN}

With the proposed configuration shown in Fig. 1, the ES is generated at both nozzles connected with electrodes of a high voltage source. As the system is primarily disconnected from ground, it yields a chargebalance with simultaneous charge neutralization, which is discussed latter below. Different from the traditional multi-electrosprays in which each ES is powered by a different voltage, the present bipolar electrostatic atomization (BESA) is designed with two nozzles, each plays the role as both the spray and reference electrodes for each other and then they define an electric field.

In the new configuration, the electric field is bended outward the electrode axes as depicted in Fig.1. In order to achieve this effect, chamfered nozzle tips are designed to initially direct the spraying. Each chamfered tip creates a stronger electric field and a large effective meniscus with radius $r$, i.e. lower capillary pressure. This effectively facilitates the starting condition for the formation of ES as stated by the Taylor cone $\varepsilon_{0} E^{2} / 2>$ $\sigma_{s} / r$. In other words, a relevant chamfer angle at the capillary tip plays a vital role to direct the initial spraying and also to reduce the initial voltage for the start-up of spraying.

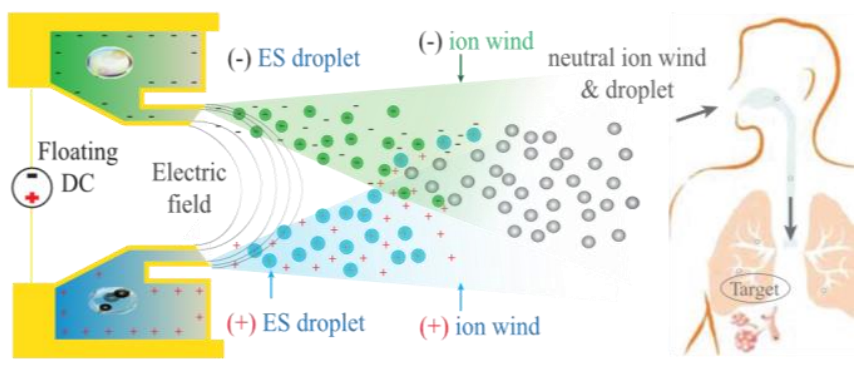

Figure 1. Mechanism of the proposed BESA approach with chambered nozzle tips.

After being generated at the vicinity of the electrode tips, two clouds of charged particles gain an initial momentum to move away along the direction parallel with the electrode axes. The electric field controls the two ion clouds of opposing polarities to impinge each other inside the area between the spray electrodes but not to let them reach their counter electrodes. Owing to the gained 
momentum, the cloud of ions moves forward to form a flow of particles. Thus, the present symmetrical configuration allows optimize the droplet migration away from the electrodes along with the electrodes' axis. It can be noted that the present bipolar electrospray is a new conceptual design where the electrodes' tips of the same curvature generate the corona discharge to form the electrospray. Therefore, the spray plume would be composed of both ionic air and atomized particles which impinge and neutralize each other. Since the generated ionic cloud of air by the corona discharge at two electrodes has high recombination rate, the neutralizing process occurs quickly and effectively to cut-off the amount of net charge [5][6].

\section{MULTI-PHYSICS SIMULATION \\ ELECTROSTATIC ATOMIZATION}

OF

The generation of liquid cone and jet are simulated using computational fluid dynamic model. Inside the liquid, the electric field induces electric body forces concentrated at the surface meniscus and tracked by a level set function, where $\phi=1$ and 0 are for the liquid and the surrounding air, respectively. Assuming liquid is linear dielectric and incompressible, the motion of liquid is governed by the continuity and momentum conservation equations as follows.

$$
\begin{aligned}
& \frac{\partial \phi}{\partial t}+\vec{u} \cdot \nabla \phi=\nabla \cdot(\nabla \phi-\phi(1-\phi)) \frac{\nabla \phi}{|\nabla \phi|} \\
& \nabla \cdot \vec{u}=0 \\
& \rho\left[\frac{d \vec{u}}{d t}+(\vec{u} \cdot \nabla) \vec{u}\right]=-\nabla p+\eta \nabla^{2} \vec{u}+\overrightarrow{\sigma_{s}}+\overrightarrow{f_{e}}+\rho \vec{g}
\end{aligned}
$$

where $p$ is the pressure, $\vec{u}$ the fluid velocity, $\overrightarrow{\sigma_{s}}$ the body force due to the surface tension and $\vec{g}$ the gravitational constant. The electric force $\overrightarrow{f_{e}}$ is assumed as $\overrightarrow{f_{e}}=q \vec{E}$, where $q$ is the charge density and $\vec{E}$ the electric field. The charge density is determined by Gauss's law as $q=$ $\varepsilon \varepsilon_{0} \nabla \cdot \vec{E}=-\varepsilon \varepsilon_{0} \nabla^{2} V$, where $\varepsilon$ is the relative dielectric constant of the liquid, $\varepsilon_{0}=8.85 \mathrm{pF} / \mathrm{m}$ the permittivity of vacuum and $V$ the electric potential. The electric body force can be expressed as:

$$
\overrightarrow{f_{e}}=-\left(\varepsilon \varepsilon_{0} \nabla^{2} V\right) \vec{E}
$$

In addition, the charge conservation is described as:

$$
\frac{d q}{d t}=\nabla \cdot \vec{J}=q \vec{u}+\sigma \vec{E}
$$

where $\sigma$ is the electrical conductivity of liquid.

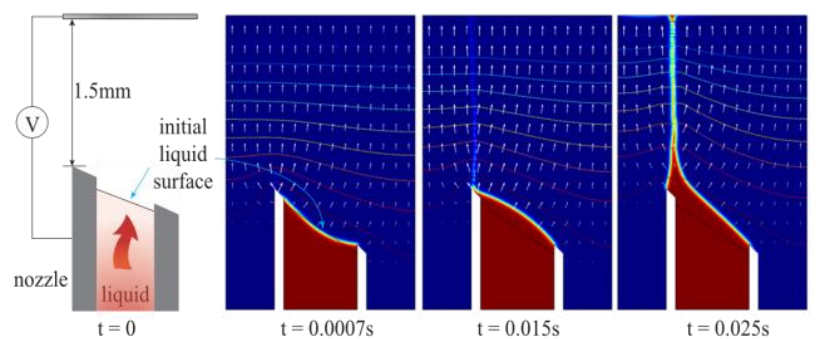

Figure 2. Transient simulation of the proposed BESA: model (left), and simulation results of liquid meniscus movement, potential distribution (contour lines) and electric field (white arrows) at different times.

The nozzle with a chamfer angle of $60^{\circ}$ at its tip is applied at a voltage of $2.3 \mathrm{kV}$. For simplicity, the ground electrode is placed normal to the nozzle axis at the distance of $1.5 \mathrm{~mm}$ from the nozzle tip. The specifications of working liquid include surface tension $\sigma_{s}=28 \mathrm{mN} / \mathrm{m}$, density $\rho=0.9 \mathrm{~g} / \mathrm{ml}$, viscosity $\mu=6 \mathrm{mPas}$ and relative permittivity $\varepsilon=20$. The flow-rate of injection, given by $Q \sim \sigma_{s} \varepsilon \varepsilon_{0} / \rho \sigma$, is set at $5 \mathrm{ml} / \mathrm{h}$ into the nozzle. The numerical model and results are presented in Figure 2.

Due to wettability, liquid moves to the tip of the nozzle in a time period of $0.0007 \mathrm{~s}$. With a sufficiently strong electric field acting on the liquid surface at the capillary tip, the charge density induced on the surface yields a radial electrostatic pressure equilibrated with the surface tension. A sharp chamfer angle tip of the nozzle yields the natural instability of the meniscus, and then a singularity that will be subsequently multiplied with the concentration of electric field at $t=0.015 \mathrm{~s}$ in Fig. 2. By the electric field, the critical volume of liquid droplet before being teared off from the capillary tip decreases and the droplet is deformed into a conical shape, yielding a generated jet from chamfered tip as expected as we can see at $\mathrm{t}=0.025 \mathrm{~s}$.

\section{EXPERIMENT AND RESULTS}

A $10 \mathrm{~mm} \times 10 \mathrm{~mm}$ microchip sample was fabricated by microfabrication technique. A $400 \mu \mathrm{m}$ silicon wafer was dry-etched from the top to form two liquid chamber liked wells, each is connected to one nozzle with the same depth of $130 \mu \mathrm{m}$. A similar dry-etching process is conducted from the bottom to release the free-standing nozzle. That prevents liquid leak to the substrate. The distance between two nozzles is $2 \mathrm{~mm}$ and the two nozzle tips are chamfered as designed. The chip is coated by a thin gold layer to increase electrical conductivity between the on-chip and off-chip features. The fabricated device is soldered on printed circuit board and the bridge between two liquid wells are finally removed to achieve two independent and symmetrical chamber-nozzle sets as seen in Fig.3.

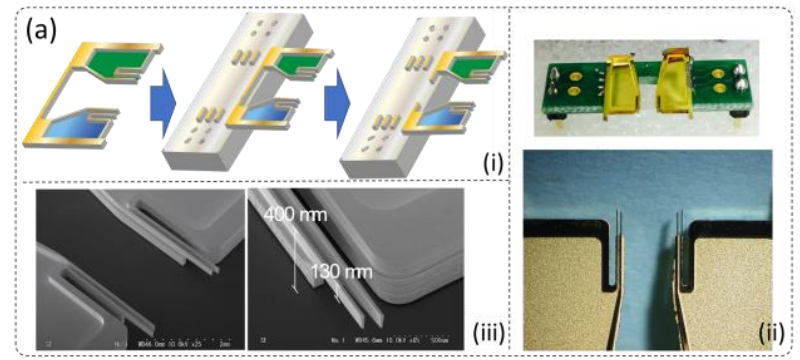

Figure 3. Micro-droplet by on-chip electro-hydrodynamic using the developed approach: a schema of the fabrication process and packaged system.

Two electrodes of a high-voltage generator is connected to each chamber. A closed-loop control system is set-up to maintain a desired discharge current sensed at the negative electrode using an adjustable current unit. The discharge current is recorded at the negative electrode by a shunt-resistor and a built-in comparator. An isolated power unit (battery) is used to ensure that the current measured at the negative electrode which represents the 
creation of the negative charge and is considered as the mirror image of the current at the positive polarity for the positive charge. The two nozzles/electrodes are strictly isolated from each other by conformal coating (Shinetsu) which also prevents leaking of liquid between two chambers.

A mixed solution of polyethylene glycol (PEG 200, average mol. wt. 200) and dipropyleneglycolmonomethyl ether (DPM, Sigma-Aldrich 99\%) with a volumetric ratio of $5 \%$ is used to investigate and evaluate droplets of the spraying. Additionally, to investigate the capability of device in delivering liquid and/or solid particles, polystyrene particles of $1.0 \mu \mathrm{m}$ diameter are introduced into the working solution. The solution is introduced into two wells using a micro dispenser. The whole electrospray is observed using a digital microscope (Dinolite EDGETM).

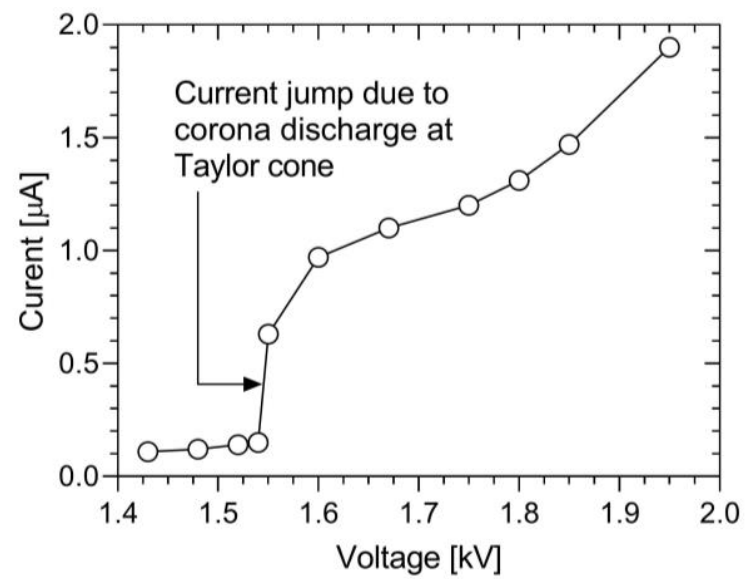

Figure 4. I-V Characteristics (I-V) of the present system for each nozzle: Connecting one nozzle with a positive electrode and another one with the negative electrode of power source while plate is grounded.

The current \& voltage (I-V) characteristics of the system is experimentally determined for a range of applied voltages which gradually increase as shown in Fig. 4. Experimental observations show that at the two nozzles, the electrospray starts at $1.34 \mathrm{kV}$ with the corresponding spray current of $0.11 \mu \mathrm{A}$. This current linearly increases to $0.15 \mu \mathrm{A}$ at voltage of $1.54 \mathrm{kV}$. Over this onset value, the currents strongly step up when the voltage continues increasing, dramatically jump to 0.97 $\mu \mathrm{A}$ when voltage reaches $1.56 \mathrm{kV}$. The current jump indicates that the corona discharge started at nozzle tip when a large number of air ions gained enough momentum to move through the inter-electrode space and ionic winds then move into the device. These ionic winds will neutralize the flow of sprayed droplet of the opposite charge and efficiently drives the droplets away from both electrodes [7]. It is worth noting that the I-V curve after the current jump does not fully agree with the relation $V \propto \sqrt{I}$ that has been found in dual ionic wind [8]. This can be justified by the co-existence of ionic wind \& electrospray which causes a small change in the I-V relation. This co-existence could improve the stability of the Taylor cone, preventing the transition from the single to multiple jet modes. The current then continues increasing with the increase of the voltage which can be used to control the electrospray regime.

The suspension solution of polystyrene is directly sprayed onto a glass substrate at a distance of $15.0 \mathrm{~cm}$ from the nozzle. The substrate is pre-covered by deionized water to catch liquid droplets but not to impact their shape. An inversed digital microscope (Olympus IX71) is used to measure the size of collected particles on the substrate. Experimental observation by Fig. 5.a depicts the appearing of droplets (Right-inset of Fig. 5a) and polystyrene particles of $1 \mu \mathrm{m}$ (Left-inset of Fig. 5a) on the substrate. Polystyrene particles with a white center are brightened than droplets.

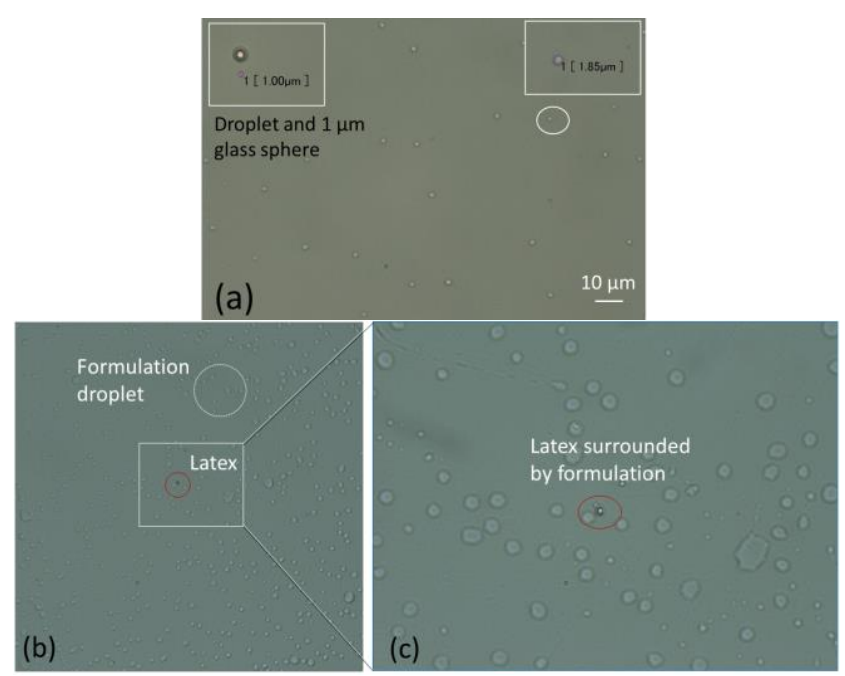

Figure 5. Experimental results of electro-spraying the generated droplets and polystyrene particles: (a) droplets of the solution PEG-DPM and polystyrene particles collected on DI-covered silica glass substrate: Rightinset shows a liquid droplet and Left-inset shows a polystyrene core droplet; and (b)\&(c) optical images and of sprayed droplets collected on a dry glass plate.

Another testing was also carried out where nanoparticles are gathered after being evaporated in-flight (Fig. 5b). For this testing, the present BESA device is connected to one side of plastic tube whereas another side is linked to a vacuum pump with a flowrate of 1.0 litre/min to extract particles. Particles were collected on an atomically flat glass substrate (Sigma Aldric) located at one end of the tube. This set-up allows neutralised particles travel a longer distance before being collected on the substrate meanwhile the charged particles are attracted and kept on the wall of the tube but not to reach to the substrate.

The distribution of droplet sizes by the present electrospray was considered and analyzed using an aerosol spectrometer. Experimental observations recorded by Fig. 6 show that the droplet size covers on a range from $0.5 \mu \mathrm{m}$ to $2.5 \mu \mathrm{m}$, the droplets' size varies around $2.0 \pm 0.5 \mu \mathrm{m}$. The experiment was independently repeated in three times whose size distribution of particles are almost the same as shown in Fig. 6. That demonstrates that the charge reduced particles are efficiently generated and delivered. It is worth noting that there is not significant difference when spraying working solutions 
with and without solid polystyrene particles as shown in Fig. 6b.
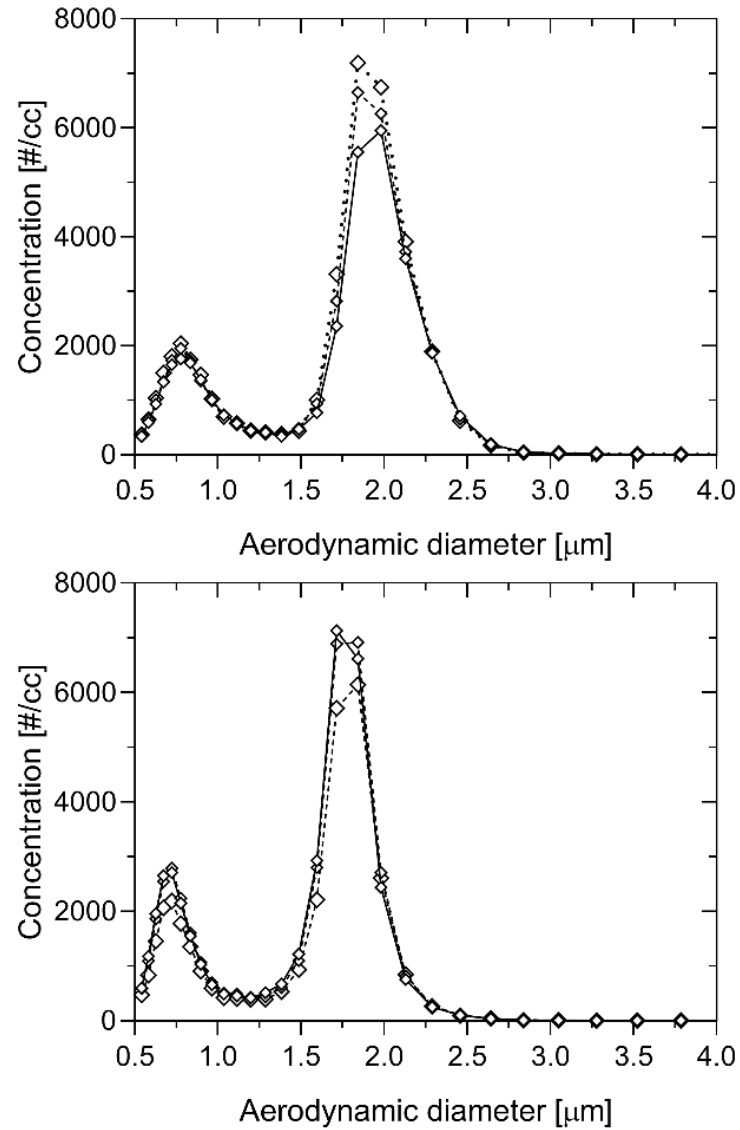

Figure 6. Distribution of droplet size generated by the developed BESA at a current of $1.2 \mu A$ with liquid solution (Top-Figure) and with polystyrene suspended solution (Bottom-Figure). Polystyrene particles of $1 \mu \mathrm{m}$ diameter. The experiment was independently repeated in three times.

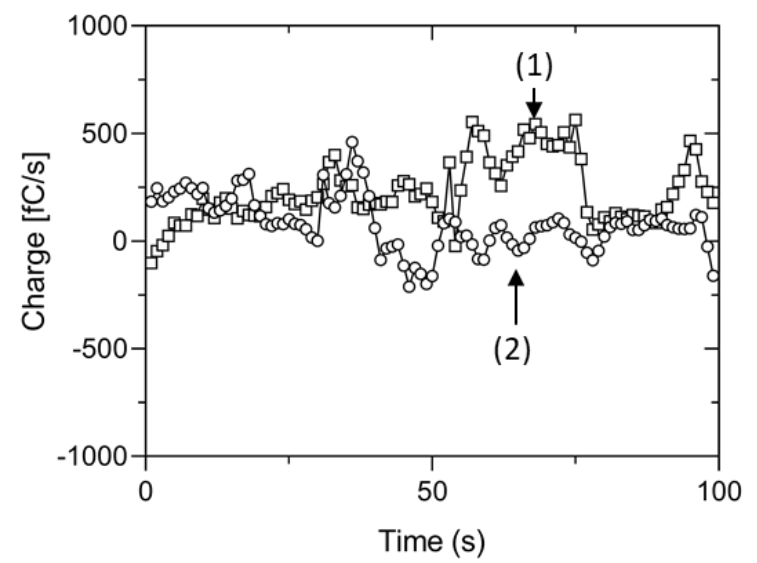

Figure 7. The charge of droplets by the present electrospray method measured by an electrometer for two cases (1) solution of PEG and DPM; and (2) polystyrene suspended solution of PED \& DPM.

In order to investigate the charge density of spayed particles by the present method, an electrometer probe is installed at the outlet of the tube. Results by Fig. 8 depict the maximum charge density of particles is approximately
$100 \mathrm{fA}$ for both cases of the liquid solution and the suspension with polystyrene particles. This very low net charge is insignificant in comparison with the discharge current at the order of microamperes as shown in Fig. 4. In other words, owing to the balance/elimination of the positive and negative charged particles, the device can generate fundamentally neutral particles.

\section{CONCLUSIONS}

A new conceptual design of electrospray together with its prototype have been developed to demonstrate the capability of spaying viable, safe, neutral and alternative droplets. The approach can be applied in micro/nanoencapsulation, bio-scaffold production as well as polymeric micro/nanoparticle fabrication over conventional fabrication techniques as well as DC electrospraying/electrospinning. Several initial experimental results by the work shows the potential application of the present device in drug delivery.

\section{REFERENCES}

[1] Q. T. Zhou, P. Tang, S. S. Y. Leung, J. G. Y. Chan, and H. K. Chan, "Emerging inhalation aerosol devices and strategies: Where are we headed?," Advanced Drug Delivery Reviews, vol. 75, pp. 3-17, 2014, doi: 10.1016/j.addr.2014.03.006.

[2] I. M. El-Sherbiny, N. M. El-Baz, and M. H. Yacoub, "Inhaled nano- and microparticles for drug delivery," Global Cardiology Science and Practice, vol. 2015, no. 1, p. 2, 2015, doi: 10.5339/gcsp.2015.2.

[3] V.T. Dau, T.-K. Nguyen, D.V. Dao, Charge reduced nanoparticles by sub-kHz ac electrohydrodynamic atomization toward drug delivery applications, Appl. Phys. Lett. 116 (2020) 023703.

[4] J. Fernandez de la Mora and C. Barrios-Collado, Aerosol Sci. Technol. 51(6), 778-786 (2017)

[5] B. M. Smirnov and H. S. W. M. Sir., Negative ions. 1982.

[6] V. T. Dau, T. X. Dinh, C.-D. Tran, T. Terebessy, T. C. Duc, and T. T. Bui, "Particle precipitation by bipolar corona discharge ion winds," J. Aerosol Sci., vol. 124, no. December 2017, pp. 83-94, Oct. 2018.

[7] V. T. Dau, T. X. Dinh, T. Terebessy, and T. T. Bui, "Bipolar corona discharge based air flow generation with low net charge," Sensors and Actuators A: Physical, vol. 244, pp. 146-155, 2016, doi: 10.1016/j.sna.2016.03.028.

[8] V. T. Dau, C. D. Tran, T. X. Dinh, L. B. Dang, T. Terebessy, and T. T. Bui, "Estimating the effect of asymmetric electrodes in bipolar discharge ion wind generator," IEEE Transactions on Dielectrics and Electrical Insulation, vol. 25, no. 3, pp. 900-907, Jun. 2018, doi: 10.1109/TDEI.2018.006881.

\section{CONTACT}

*V. T. Dau, tel: +61755529723; v.dau@griffith.edu.au *Tung T. Bui, tel: +842437547709; tungbt@vnu.edu.vn 